\title{
Functionalized milk-protein-coated magnetic nanoparticles for MRI-monitored targeted therapy of pancreatic cancer
}

This article was published in the following Dove Press journal:

International Journal of Nanomedicine

7 July 2016

Number of times this article has been viewed

\author{
Jing Huang ${ }^{1,2}$ \\ Weiping Qian ${ }^{3}$ \\ Liya Wang ${ }^{1,2}$ \\ Hui Wu' \\ Hongyu Zhou ${ }^{3}$ \\ Andrew Yongqiang Wang ${ }^{4}$ \\ Hongbo Chen ${ }^{5}$ \\ Lily Yang ${ }^{3}$ \\ Hui Mao ${ }^{1,2}$ \\ 'Department of Radiology and \\ Imaging Sciences, ${ }^{2}$ Center for Systems \\ Imaging, ${ }^{3}$ Department of Surgery, \\ Emory University School of Medicine, \\ Atlanta, GA, USA; ${ }^{4}$ Ocean Nanotech \\ LLC, Springdale, AR, USA; ${ }^{5}$ School \\ of Life and Environmental Sciences, \\ Guilin University of Electronic \\ Technology, Guilin, Guangxi, People's \\ Republic of China
}

Correspondence: Hui Mao

Department of Radiology and Imaging Sciences, Emory University School of

Medicine, I84I Clifton Road, Atlanta,

GA 30329, USA

$\mathrm{Tel}+$ I 4047120357

Fax + I 4047125689

Email hmao@emory.edu

Lily Yang

Department of Surgery, Emory University School of Medicine, 1365 Clifton Road,

Atlanta, GA 30329, USA

Tel +l 4047784564

Fax + I 404 7I25689

Email lyang02@emory.edu
Abstract: Engineered nanocarriers have emerged as a promising platform for cancer therapy. However, the therapeutic efficacy is limited by low drug loading efficiency, poor passive targeting to tumors, and severe systemic side effects. Herein, we report a new class of nanoconstructs based on milk protein (casein)-coated magnetic iron oxide (CNIO) nanoparticles for targeted and image-guided pancreatic cancer treatment. The tumor-targeting amino-terminal fragment (ATF) of urokinase plasminogen activator and the antitumor drug cisplatin (CDDP) were engineered on this nanoplatform. High drug loading $(\sim 25 \mathrm{wt} \%)$ and sustained release at physiological conditions were achieved through the exchange and encapsulation strategy. These ATF-CNIO-CDDP nanoparticles demonstrated actively targeted delivery of CDDP to orthotopic pancreatic tumors in mice. The effective accumulation and distribution of ATF-CNIO-CDDP was evidenced by magnetic resonance imaging, based on the $\mathrm{T}_{2}$-weighted contrast resulting from the specific accumulation of ATF-CNIO-CDDP in the tumor. Actively targeted delivery of ATF-CNIO-CDDP led to improved therapeutic efficacy in comparison with free CDDP and nontargeted CNIO-CDDP treatment. Meanwhile, less systemic side effects were observed in the nanocarrier-treated groups than that in the group treated with free CDDP. Hematoxylin and Eosin and Sirius Red staining of tumor sections revealed the possible disruption of stroma during the treatment with ATF-CNIO-CDDP. Overall, our results suggest that ATF-CNIO-CDDP can be an effective theranostic platform for active targeting-enhanced and image-guided cancer treatment while simultaneously reducing the systemic toxicity.

Keywords: active targeting, drug delivery, cisplatin, magnetic nanoparticles, magnetic resonance imaging, pancreatic cancer

\section{Introduction}

Pancreatic cancer is the fourth leading cause of cancer deaths in the United States. ${ }^{1,2}$ Although significant discoveries and advances in cancer detection and therapeutic approaches have been made in the past decades, the prognosis of pancreatic cancer patients remains poor with a 5 -year survival rate of only $\sim 6 \%$. $^{2}$ Currently, chemotherapy and radiotherapy are the major treatment options for pancreatic cancer patients, as $85 \%$ of patients are diagnosed at the advanced stage. ${ }^{2}$ However, low specificity of chemotherapy drugs, poor efficiency in drug delivery, and drug resistance by cancer cells limit the treatment efficacy. Clinical trials of the combination chemotherapy with targeted therapy or radiation therapy are being conducted. Among them, several showed a statistically significant improvement in survival. ${ }^{3-5}$ For example, the FOLFIRINOX regimen, composed of fluorouracil (5-FU), leucovorin, irinotecan, and oxaliplatin, has resulted in 4 months longer survival than the standard 
treatment with gemcitabine alone. ${ }^{3}$ However, severe side effects associated with the FOLFIRINOX regimen limit its application in pancreatic cancer patients. Cisplatin (also called cis-diamminedichloroplatinum (II) [CDDP]) is a potent anticancer chemotherapy drug that can intercalate into the double-stranded DNA in cancer cells. Despite its high potency, CDDP is only considered as an adjunct drug in pancreatic cancer treatment, usually combined with gemcitabine, because of the side effects and low efficiency caused by its fast clearance, leading to a lack of targeting and inactivation of drug during the process. ${ }^{6}$

Recent advances in nanomaterials and nanotechnology have presented a number of promising platforms to improve the specificity and efficiency of cancer drug delivery. Lipid- or polymer-based nanoparticle delivery methods/materials and cancer-cell-targeted delivery have been studied for improving treatment by reducing side effects and enhancing drug accumulation at the tumor site using pancreatic cancer cell lines and human pancreatic tumor xenograft models., ${ }^{5,-12}$ These studies demonstrated promising strategies to use nanotechnology for delivery of cancer therapeutics, but also suggested that new strategies are needed for developing stable nanocarriers with prolonged circulation time and target-specific delivery and for lowering the drug resistance of cancer cells. ${ }^{13-15}$ In addition, noninvasive monitoring of drug delivery to the tumor site is desirable to optimize delivery in order to improve the treatment efficacy. ${ }^{16}$

Herein, we report a new targeted drug delivery nanosystem based on milk protein (casein [CN])-coated magnetic iron oxide (CNIO) nanoparticles, composed of a cancer cell targeting moiety that is the amino-terminal fragment (ATF) of urokinase plasminogen activator with an affinity to urokinase plasminogen activator receptor (uPAR), and the anticancer agent or CDDP for pancreatic cancer treatment. CNIO nanoparticles is not only an excellent magnetic resonance imaging (MRI) probe as previously reported, ${ }^{17,18}$ but is also capable of loading a sufficient amount of CDDP in the $\mathrm{CN}$ layer through the ligand exchange of chloride groups in CDDP with the carboxyl groups in the CN protein. ${ }^{19}$ The coating layer also renders sustained CDDP release, allowing sustained drug concentrations during circulation as well as targeted delivery and reduction of side effects due to the rapid release to the normal organs. Our result revealed that the developed ATF-CNIO-CDDP exhibited specific targeting to uPAR-overexpressing pancreatic cancer cells. Furthermore, ATF-CNIO-CDDP demonstrated targeted delivery of CDDP and improved therapeutic effect in an orthotopic pancreatic cancer model.

\section{Materials and methods Materials}

All chemicals, including CDDP, were purchased from SigmaAldrich (St Louis, MO, USA) unless otherwise specified, and were used as received. 1-Ethyl-3-[3-dimethylaminopropyl]carbodiimide hydrochloride (EDC) and $N$-hydroxysulfosuccinimide (Sulfo-NHS) were obtained from Thermo Scientific (Rockford, IL, USA). All cell culture materials (media and supplements) were purchased from Invitrogen (Burlington, ON, USA). Ultra-4 centrifuge tubes (100 kDa MWCO) were purchased from Millipore (Billerica, MA, USA).

\section{Preparation of ATF-CNIO-CDDP nanoparticles}

First, CNIO nanoparticles were prepared as described previously. ${ }^{17}$ Human ATF peptide of the receptor-binding domain of uPA was prepared according to the established protocol. ${ }^{20}$ The ATF peptide was then conjugated to $\mathrm{CNIO}$ nanoparticles through EDC/NHS cross-link. Briefly, ATF $(0.5 \mathrm{mg})$ was reacted with $\mathrm{N}$-hydroxysuccinimide (NHS, $0.1 \mathrm{mg}$ ) and 1-ethyl-3-(3-dimethylaminopropyl)-carbodiimide (EDAC, $0.2 \mathrm{mg}$ ) in 2-(N-morpholino)ethanesulfonic acid (MES) buffer solution ( $1 \mathrm{M}, \mathrm{pH} 5.5$ ) for 10 minutes, and then mixed with CNIO nanoparticles in borate buffer solution $(0.5 \mathrm{M}$, $\mathrm{pH} 8.5)$. After reaction for 2 hours, the product was collected by ultracentrifugation (100 kDa MWCO) and washed with deionized water. For drug loading, CDDP was mixed with $\mathrm{CNIO}$ nanoparticles in $50 \mathrm{mM} \mathrm{NaCl}$ solution at $4^{\circ} \mathrm{C}$ overnight. Free CDDP was removed by ultracentrifugation (100 kDa MWCO) followed by washing three times with deionized water.

\section{Characterization of ATF-CNIO-CDDP nanoparticles}

The size and morphology of the prepared ATF-CNIO-CDDP nanoparticles were determined by transmission electron microscope (TEM, Hitachi H-7500 [Hitachi Ltd, Tokyo, Japan], with an accelerating voltage $75 \mathrm{kV}$ ). The average hydrodynamic sizes and zeta potentials of different nanoparticles were determined using dynamic light scattering (DLS; Malvern Zetasizer Nano S-90; Malvern Instruments, Malvern, UK) equipment. To quantify the amount of CDDP and iron, selected samples were dissolved in concentrated nitric acid, then carefully diluted, and finally $\mathrm{pH}$ adjusted. Pt concentration was measured by colorimetric method with $o$-phenylenediamine (OPDA), ${ }^{21}$ while Fe concentration was determined by the phenanthroline colorimetric method. ${ }^{17}$ The percentage of CDDP loaded on CNIO nanoparticles was 
calculated as the ratio of the amount of CDDP loaded on the CNIO nanoparticles to Fe based on the weight, ie, CDDP loading $\%=$ (weight of CDDP in nanoparticles/weight of $\mathrm{Fe}$ in nanoparticles) $\times 100 \%$.

\section{Measurement of CDDP release from ATF-CNIO-CDDP}

To measure the amount of CDDP released from the nanoparticles, the ATF-CNIO-CDDP solution at a concentration equivalent to $60 \mu \mathrm{g} / \mathrm{mL}$ free CDDP (or $200 \mu \mathrm{M}$ ) was diluted and $\mathrm{pH}$ adjusted to $\mathrm{pH} 5.5$ or 7.4 , and then incubated at $37^{\circ} \mathrm{C}$. At each predetermined time point and interval, a portion of the mixture was collected and centrifuged with an ultra-4 centrifuge tube (cut-off size $100 \mathrm{kDa}$ ). The supernatant was collected, and the same amount of fresh buffer solution was added. The CDDP content in the supernatant was then determined by a colorimetric method. ${ }^{21}$ Briefly, $500 \mu \mathrm{L}$ of a CDDP sample solution was mixed with the same volume of OPDA solution in dimethylformamide $(1.4 \mathrm{mg} / \mathrm{mL})$. The mixture was heated to $100^{\circ} \mathrm{C}$ for 10 minutes, followed by cooling it in an ice bath at $0^{\circ} \mathrm{C}$ for 10 minutes. The absorbance of the solution was measured at 600,720 , and $800 \mathrm{~nm}$ with a microplate reader. The concentration of CDDP in the solution was obtained by calibrating against a standard curve.

\section{Determining specific binding of ATF-CNIO-CDDP to MIA PaCa-2 cells}

Ethics approval for the use of cell lines was granted by Emory University. The uPAR targeting ability of ATFCNIO-CDDP was assessed using the human pancreatic cancer MIA PaCa-2 cell line with uPAR overexpression. $\mathrm{CNIO}$ without a targeting moiety conjugated was used as the control. Cells were seeded in 8-well chamber slides at a density of $5 \times 10^{4}$ cells/well. After recovering for 24 hours, the media was replaced with that containing ATF-CNIO-CDDP at a concentration of $0.1 \mathrm{mg} \mathrm{Fe} / \mathrm{mL}$. Cells were incubated with the ATF-CNIO-CDDP-containing media at $37^{\circ} \mathrm{C}$ for an additional 4 hours. The cell monolayer was then rinsed with phosphate-buffered saline (PBS) and fixed with 4\% paraformaldehyde in the PBS solution. Prussian blue staining for iron was performed to investigate the specific binding of ATF-CNIO-CDDP or control samples to cells with different levels of uPAR expression. Briefly, freshly prepared $10 \%$ potassium ferrocyanide (II) trihydrate and $20 \% \mathrm{HCl}$ solutions were mixed and then added to wells containing fixed cells. After incubating for 1 hour, the Prussian blue staining solution was removed and the cells were washed with PBS. Counterstaining for cells was performed by adding nuclear fast red solution and incubating for 8 minutes. Stained cells were dehydrated with $70 \%$ and $100 \%$ ethanol, and then rinsed in xylene. The slides were mounted and examined under a light microscope.

\section{Assessment of anticancer efficacy in vitro}

Human pancreatic cancer MIA PaCa-2 cells were seeded in a 96-well plate at a density of $5 \times 10^{3}$ cells/well and allowed to adhere overnight. The culture medium was then replaced with medium containing free CDDP, CNIO-CDDP, or ATFCNIO-CDDP at different CDDP concentrations. After cells were cultured for another 72 hours, the cytotoxicity was determined by CCK-8 assay following the vendor-provided protocol. Briefly, cells were carefully rinsed with PBS after the drug-containing medium was removed, and this was followed by adding the CCK-8 containing medium solution. The cells were then incubated with the CCK-8 medium for 4 hours. The plate was read at the absorbance wavelength of $450 \mathrm{~nm}$ using a microplate reader (Synergy2; BioTek, Winooski, VT, USA). Cell viability was determined by comparing the absorbance of cells incubated with drugs to that of the control cells incubated without the presence of the drug.

\section{Treatment evaluation using an orthotopic pancreatic tumor model}

All animal experiments were conducted following a protocol approved by the Institutional Animal Care and Use Committee (IACUC) at Emory University. The orthotopic pancreatic cancer model was prepared by injecting MIA PaCa-2 cells into the pancreas of 8-week-old female nu/nu mice using an established surgical method. ${ }^{20}$ Mice were anesthetized by intraperitoneal injection of a ketamine:xylazine mixture (95:5 $\mathrm{mg} / \mathrm{kg}$ ) during the surgery. Incision was made on the left flank of the abdominal region where the pancreas is typically located, behind the middle of the spleen. The pancreas was then gently pulled out using forceps and $50 \mu \mathrm{L} 1 \times 10^{6}$ MIA PaCa-2 cells was carefully injected into the head of the pancreas. After injection, the pancreas was placed back in the abdominal cavity before the abdominal muscle and the skin were closed with 4-0 Polysorb sutures and surgical staples. Tumors typically reached $5 \mathrm{~mm}$ in diameter after 3 weeks and were ready for imaging and treatment experiments. Animals were randomly divided into four groups $(n=6)$ : treatment control, treated with free CDDP, treated with nontargeted CNIO-CDDP, and treated with ATF-CNIO-CDDP. The mice were treated through intravenous tail vein injection at a dose of $5 \mathrm{mg}$ CDDP $/ \mathrm{kg}$ mouse weight, while the control group received only saline injection. In total, there were four injections with 6- or 7-day intervals. The body weights were measured at each time point. At the last time point, 
the tumors were collected and weighed to determine the antitumor activity.

\section{Monitoring of drug delivery in vivo by MRI}

To investigate the delivery of ATF-CNIO-CDDP in vivo, tumor-bearing mice were scanned before and 1 week after first injection of ATF-CNIO-CDDP on a 3 T MRI scanner (Tim Trio; Siemens, Erlangen, Germany) using a $\mathrm{T}_{2}$-weighted fast spin echo sequence followed by a multi-TE fast spin echo sequence for $\mathrm{T}_{2}$ relaxometry mapping of the tumor to further validate the accumulation of nanoparticles. The MRI acquisition parameters were repetition time (TR) $=3,600 \mathrm{~ms}$, echo time $(\mathrm{TE})=12-180 \mathrm{~ms}$, flip angle $=150$, matrix $=154 \times 320$, field-of-view $(\mathrm{FOV})=70 \times 120 \mathrm{~mm}^{2}$, slice thickness $=1 \mathrm{~mm}$ and number of averages $=3$. Colorized $T_{2}$ maps were then generated as described in a previous work. ${ }^{22}$ Regions of interest were drawn surrounding the tumor to calculate the MRI signal changes or the $T_{2}$ values.

\section{Immunohistochemistry analysis}

The organs (liver, spleen, and kidney) and tumors were collected after final treatment. Prussian blue staining for the presence of iron was performed on tissues collected from the major organs and tumors following our established protocol..$^{17,22}$ Immunofluorescence labeling for UPAR was performed using a rabbit anti-uPAR monoclonal antibody, followed by Alexa Fluor 555 goat anti-rabbit IgG secondary antibody. Sirius red staining for collagen in stromal structures was performed following an established protocol. ${ }^{23}$ The stained slices were examined with a light or fluorescent microscope. For the comparison of relative collagen content, the red channel of the micrographs of Sirius-red-stained tumor sections were extracted using METLAB. Since the iron concentration is directly correlated to the $\mathrm{T}_{2}$ relaxation time measured by MRI at the given transverse relaxivity of the material, ${ }^{24}$ we used MRI relaxivity measurement to determine the relative amount of iron oxide nanoparticles accumulated in tumors collected after the imaging experiments. Briefly, tumors were homogenized in an equal weight of lysis buffer. The homogenized tissue samples were placed in the vials and then scanned on a 3 T MRI scanner using $\mathrm{T}_{2}$-weighted and multi-TE fast spin echo sequences.

\section{Statistical analysis}

Results were expressed as means \pm standard deviation when a measurement was made. When comparing two different conditions or different samples, Student $t$-tests were used to determine statistical significance. A $P$-value $<0.05$ was considered statistically significant.

\section{Results and discussion Synthesis and characterization of ATF-CNIO-CDDP}

The schematic structure of ATF-CNIO-CDDP is illustrated in Figure 1A. Bovine milk casein protein was selected as the coating material. In addition to stabilizing IO nanoparticles, casein coating provides abundant functional groups for incorporating targeting ligands on the surface. ${ }^{17} \mathrm{ATF}$, which has been previously demonstrated as an efficient targeting ligand to pancreatic tumors, ${ }^{20,25,26}$ was conjugated onto the surface using EDC/NHS via a covalent link between the carboxyl groups in ATF and the amine groups of casein coating, thus giving uPAR targeted ATF-CNIO nanoparticles. CDDP was subsequently loaded onto the particle through ligand exchange of chloride in CDDP with the carboxyl groups in $\mathrm{CN}$ protein. ${ }^{27,28}$

TEM images revealed that the magnetic iron oxide nanoparticle core with a diameter of $10.3 \pm 0.5 \mathrm{~nm}$ remained intact after surface functionalization with ATF and CDDP (Figure 1B). On the other hand, DLS measurement showed a slightly larger hydrodynamic diameter of ATF-CNIO $(26.0 \mathrm{~nm})$ compared with CNIO $(24.6 \mathrm{~nm})$, indicating the successful conjugations of targeting ligands. The amount of ATF ligands conjugated was estimated at 17 ATF molecules per particle, as determined by the Bradford assay. After loading CDDP, little change in hydrodynamic diameter was observed for ATF-CNIO-CDDP (Figure 1C). However, the surface charge shifted from -35.2 to $-31.9 \mathrm{mV}$ as the result of loading CDDP further neutralized the $\mathrm{COOH}$ groups. Using the OPDA colorimetric method, ${ }^{21}$ the amount of CDDP loaded on ATF-CNIO was determined to be 25:100 (wt) for CDDP:Fe. Compared with other early reported IO or protein-based nanoparticle carriers developed for CDDP delivery, ${ }^{28-30}$ the significantly higher CDDP loading on ATF-CNIO-CDDP can be attributed to the unique structure of casein proteins, ie, the abundant carboxyl groups together with the phosphate groups. ${ }^{31}$

To evaluate the release profile in vitro, the ATF-CNIOCDDP solutions were incubated in PBS (pH 5.5 and 7.4) at $37^{\circ} \mathrm{C}$. At both $\mathrm{pH}$ conditions, ATF-CNIO-CDDP showed sustained CDDP release (Figure 1D), with a half-life time around 10 and 4.5 hours at $\mathrm{pH} 5.5$ and 7.4, respectively. The accelerated release of CDDP at $\mathrm{pH} 5.5$ may indicate the $\mathrm{pH}$-triggered release of CDDP at low $\mathrm{pH}$ in a tumor tissue or cellular lysosome. The sustained release manner implies that most CDDP bound to casein coating through the exchange process, which could be re-exchanged by chloride or hydroxide ions after the protonation of carboxyl groups. ${ }^{31}$ 

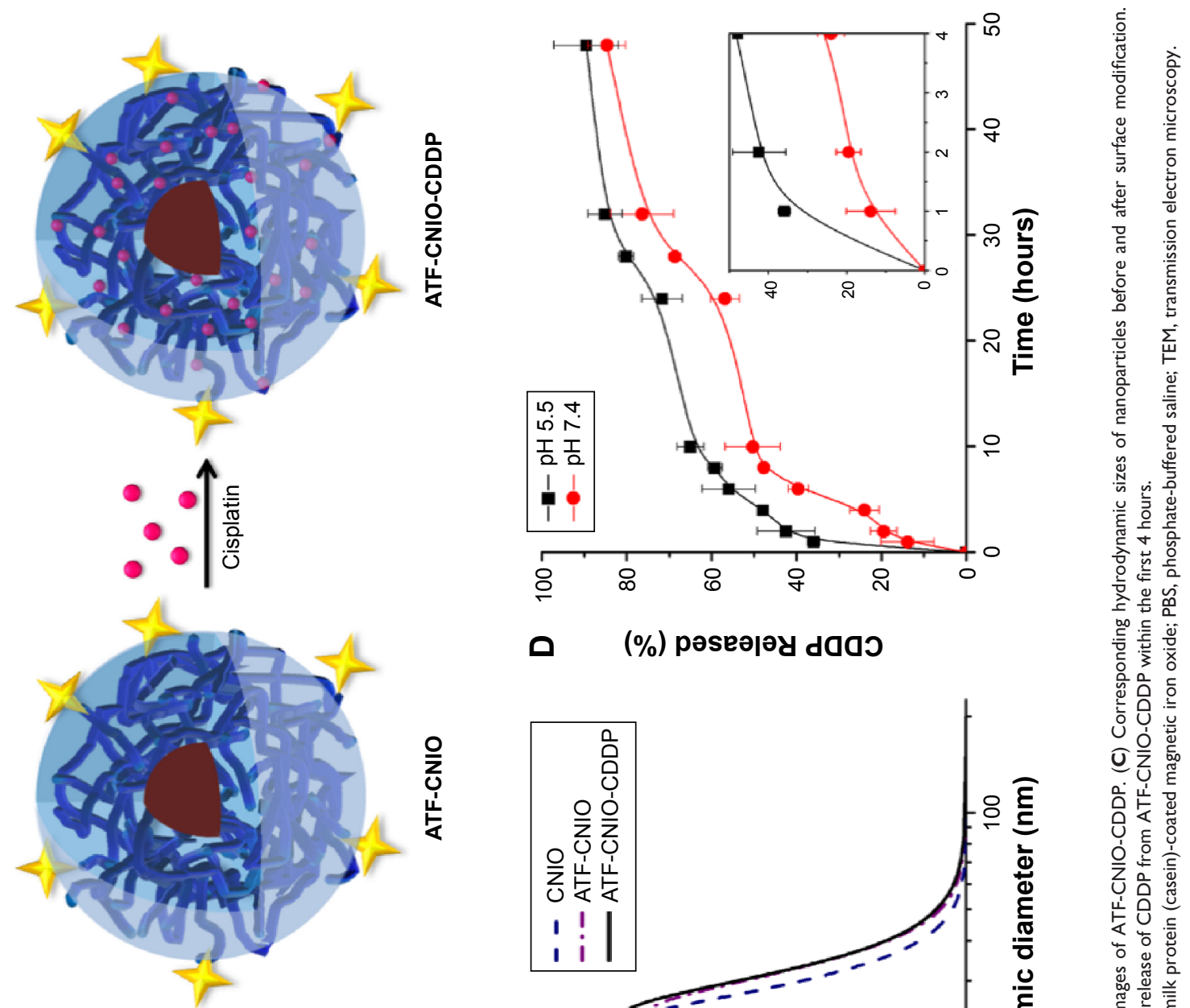

을
U⿺
这
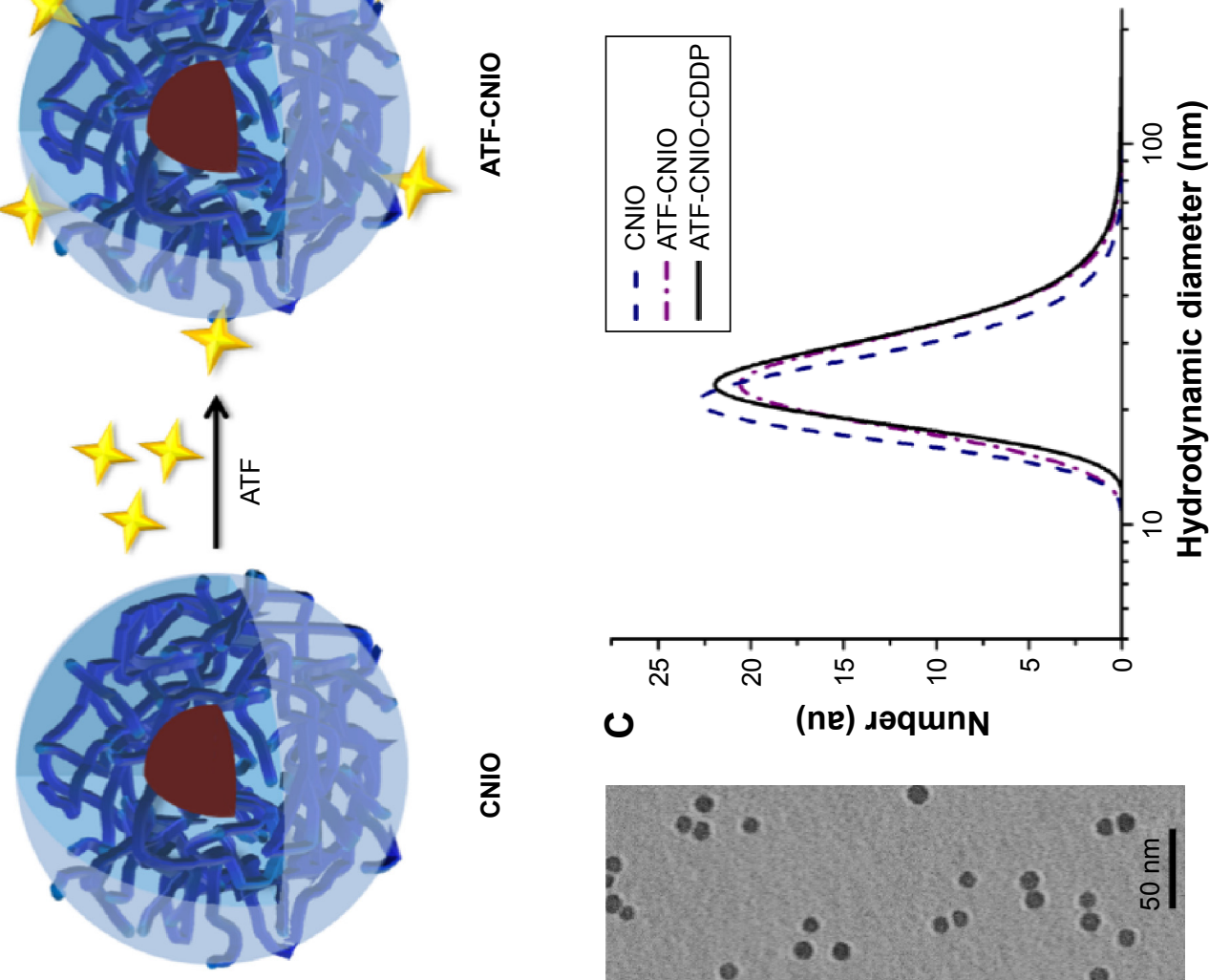

은
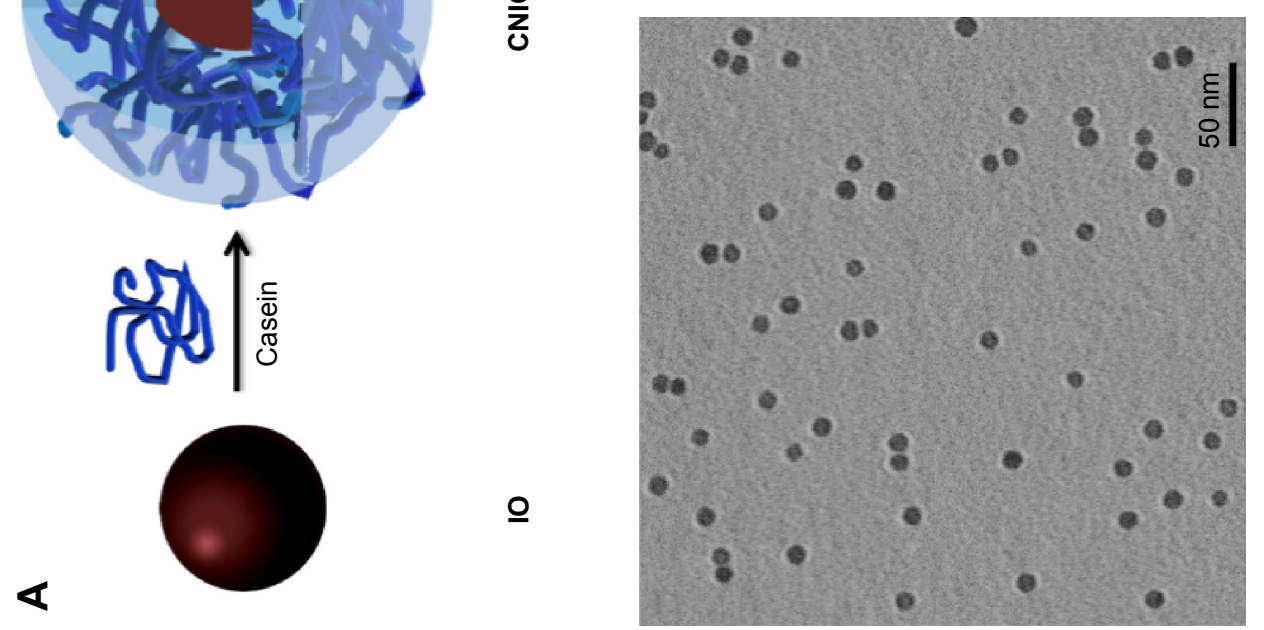

$\boldsymbol{m}$ ํํㅇ응 บิ บิ Uิ 合峞造 은 혼 诂合

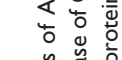

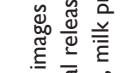

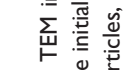
这 牙氜 记吉

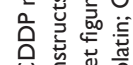

บิ

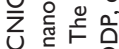
崖号宁 资产 동 ํำ

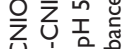

崖崖荡

《吅

记号豆

ธ

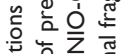

㻤记

总皆峞

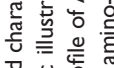

穴递立崖

号

气令这

잉

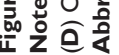




\section{Cell targeting and cytotoxicity of ATF-CNIO-CDDP}

To investigate the efficiency of uPAR-specific targeting of ATF-CNIO to uPAR-overexpressing pancreatic cancer cells, human pancreatic cancer MIA PaCa- 2 cells were incubated with ATF-CNIO and non-targeted CNIO nanoparticles (as a control) at $37^{\circ} \mathrm{C}$ for 4 hours. Subsequent Prussian blue staining for iron revealed the specific binding of ATF-CNIO to MIA PaCa-2 cells, as evidenced by strong positive blue stains (Figure 2A). No such blue stains were observed in the cells treated with nontargeted IO.

In vitro cytotoxicity of ATF-CNIO-CDDP was tested in MIA PaCa-2 cells by MTT assay. Free CDDP and nontargeted CNIO-CDDP were used as controls. Cell viability of all three groups showed dose-dependent decrease after 72 hours of treatment (Figure 2B). Both ATF-CNIO-CDDP and CNIOCDDP showed more potent cytotoxicity than free CDDP. Half-maximal inhibitory concentrations were determined to be $1.1,1.6$, and $16.1 \mu \mathrm{M}$ for ATF-CNIO-CDDP, CNIOCDDP and free CDDP, respectively. Targeted ATF-CNIOCDDP increased the in vitro treatment efficacy by 15 -fold, while the efficacy of nontargeted CNIO-CDDP increased about 10-fold over that of free CDDP. This is consistent with some previous studies that reported higher toxicity of some lipid- and polymer-formulated CDDP than free CDDP. ${ }^{12,32,33}$ The limited amount of CDDP loaded in delivery systems (eg, less than $10 \mathrm{wt} \%$ ), together with the inevitable deactivation of CDDP during their long-time preparation procedures (ie, long period of hydration), ${ }^{6}$ is usually considered as one of the major reasons for reduced therapeutic efficiency. In our case, as much as $25 \mathrm{wt} \%$ CDDP was loaded on the CNIO-CDDP delivery system, which is significantly higher than that used in other protein drug carrier formulations. ${ }^{20,28}$ In addition, drug activity is preserved by shortened preparation time and enhanced coating stability in chloride buffers.

\section{MRI monitoring of ATF-CNIO-CDDP in tumor-bearing mice}

The accumulation and distribution of drug-loaded nanoparticles at the tumor site was monitored by in vivo MRI before and 1 week after administering ATF-CNIO-CDDP. Regions of interest were drawn at the level of the maximum diameter of the tumor using the corresponding $\mathrm{T}_{2}$-weighted scans, excluding regions of pancreatic ducts, cystic lesions, and fat. Significant MRI signal intensity (SI) drops were observed in tumor areas after the administration of ATF-CNIO-CDDP, as shown in Figure 3A, resulting from the accumulation of these magnetic nanoparticles at the tumor site. The heterogeneous distribution of ATF-CNIO-CDDP reflected the tumor heterogeneity, with SI drop observed in both peripheral and central areas, indicating the efficient delivery and perfusion into tumor. The average decrease of SI in the tumor was estimated to be $\sim 31 \%$ after administration of ATF-CNIO-CDDP (Figure 3B).

\section{In vivo test of the anticancer efficacy of ATF-CNIO-CDDP}

Therapeutic efficacy of ATF-CNIO-CDDP was evaluated using orthotopic MIA $\mathrm{PaCa}-2$ pancreatic tumor-bearing
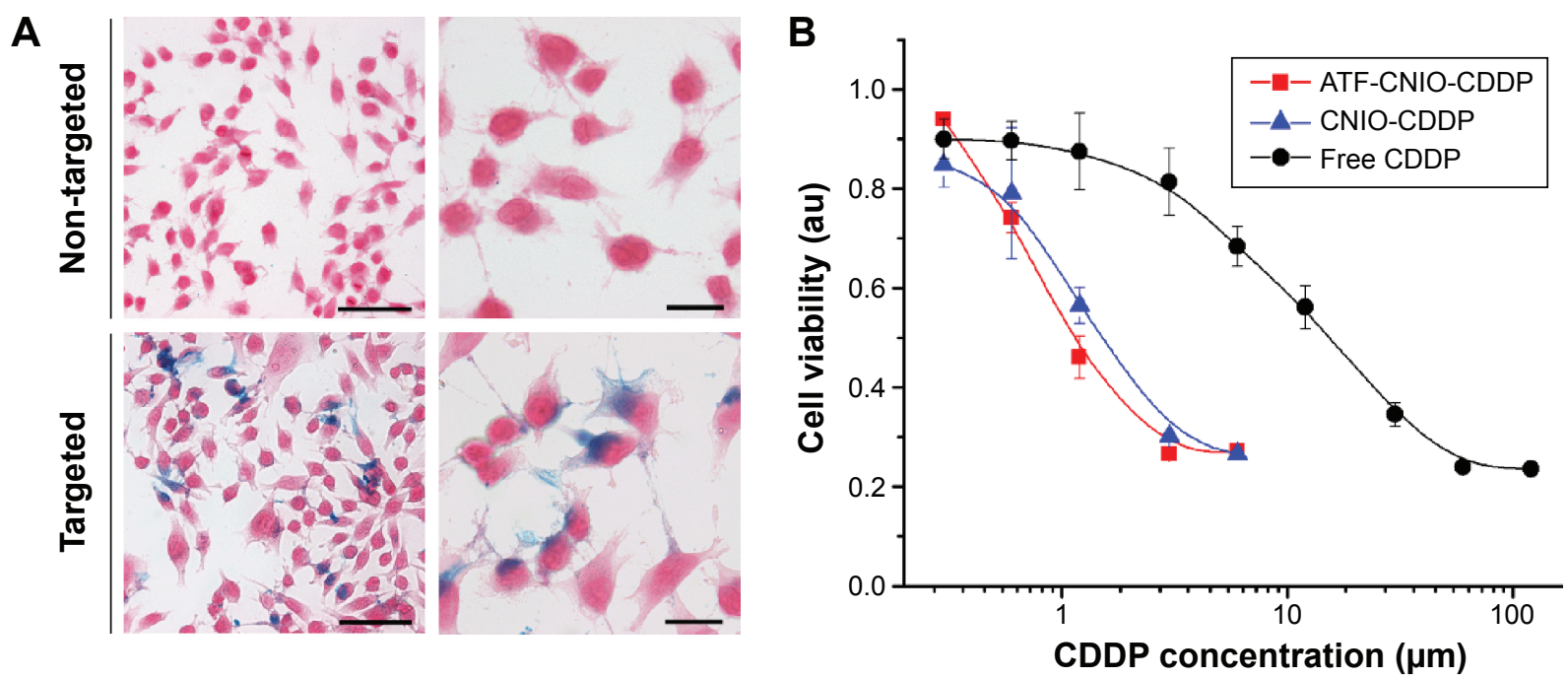

Figure 2 In vitro assay for targeting specific binding test of CNIO and ATF-CNIO nanoparticles and cellular cytotoxicity of ATF-CNIO-CDDP, CNIO-CDDP and free CDDP. Notes: (A) Representative Prussian blue staining micrographs (left) and magnifications (right) show the target specific binding of uPAR targeted ATF-CNIO comparing to nontargeted CNIO nanoparticles to human pancreatic cancer MIA PaCa-2 cells, which overexpress uPAR. Scale bars correspond to 40 and $20 \mu \mathrm{m}$ in full and magnified panels respectively. (B) In vitro cytotoxicity of ATF-CNIO-CDDP, CNIO-CDDP and free CDDP at various concentrations against MIA PaCa-2 cells measured by MTT cell proliferation assay. Abbreviations: ATF, amino-terminal fragment; au, absorbance units; CDDP, cisplatin; CNIO, milk protein (casein)-coated magnetic iron oxide; uPAR, urokinase plasminogen activator receptor. 
A

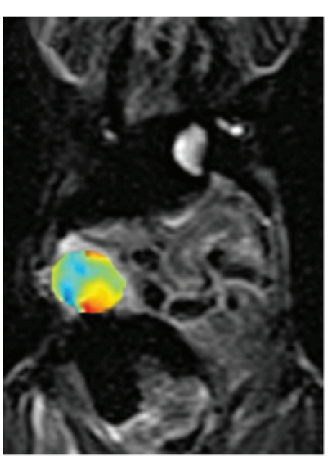

Pre

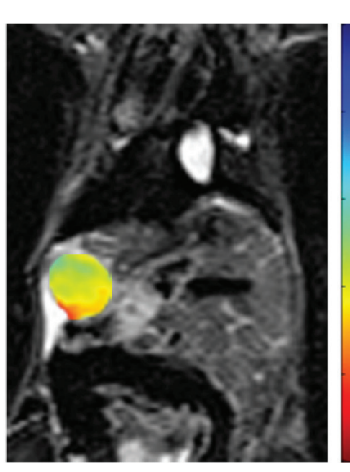

Post $1 \mathrm{wk}$

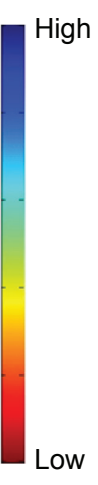

C

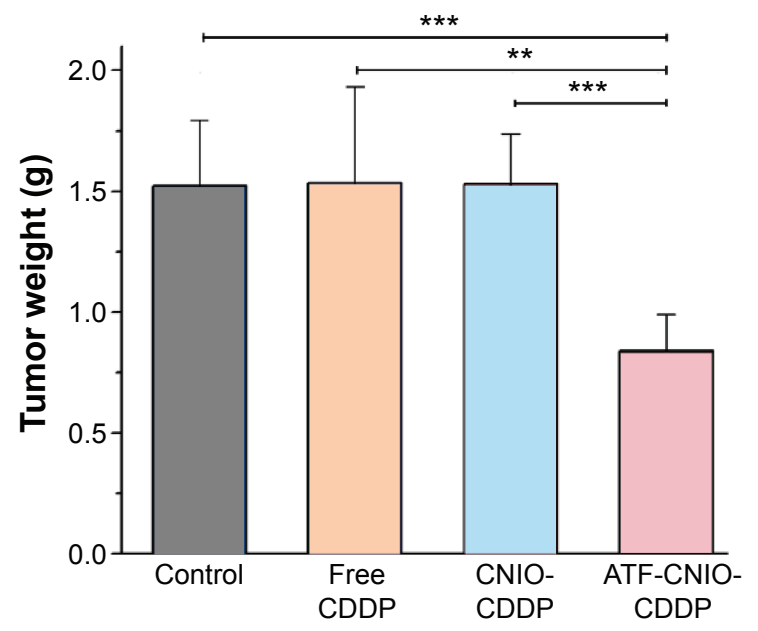

E

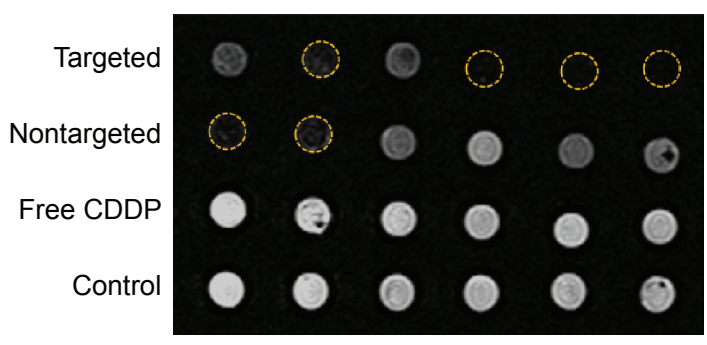

B

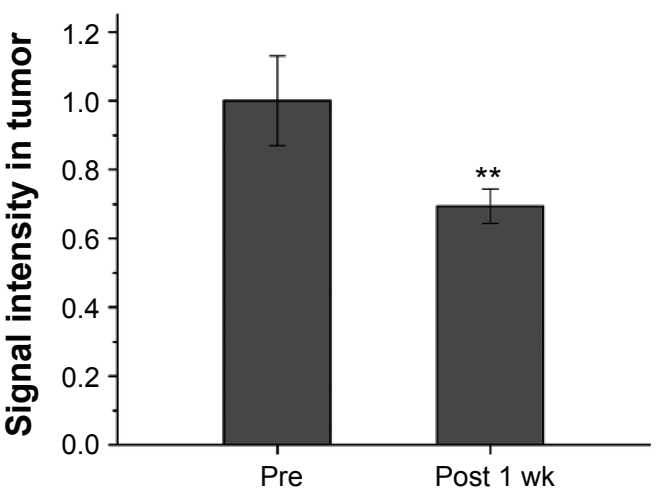

D

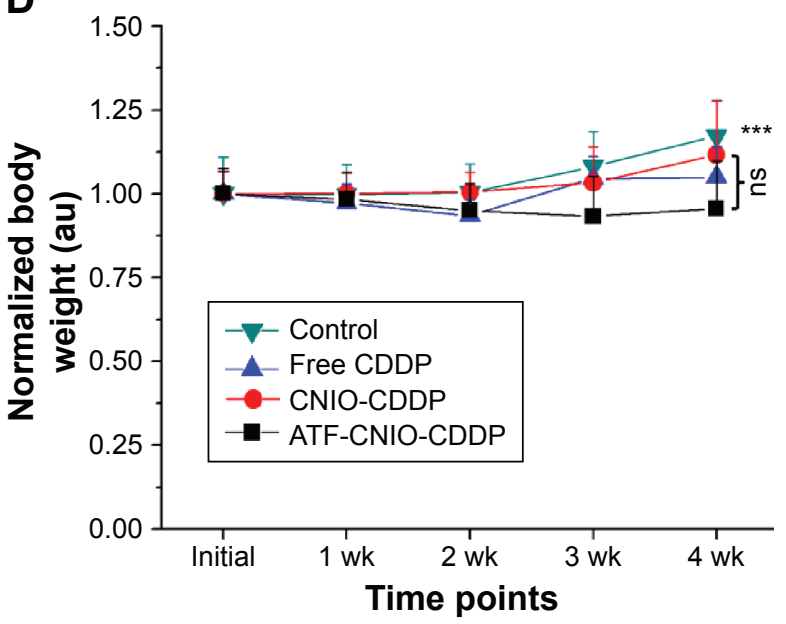

$\mathbf{F}$

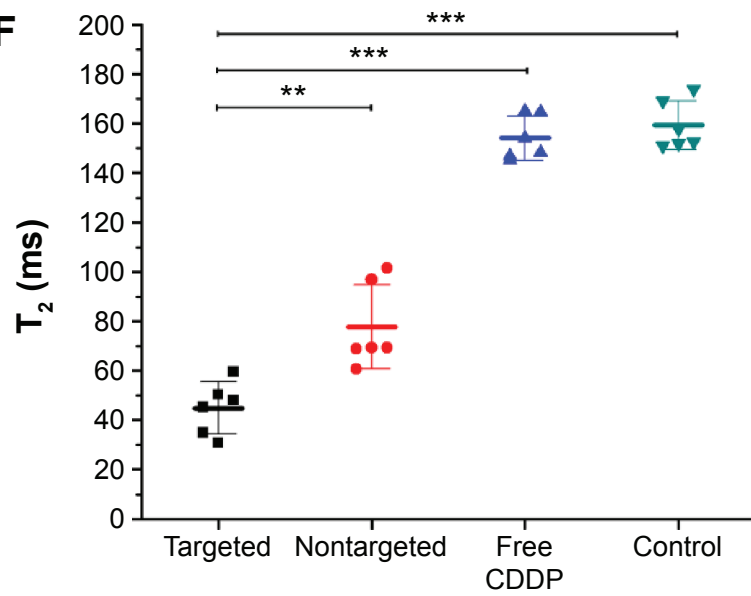

Figure 3 Treatment efficacy of ATF-CNIO-CDDP in the orthotopic pancreatic tumor model evaluated by MRI.

Notes: (A) In vivo $\mathrm{T}_{2}$-weighted MRI of a mouse bearing orthotopic pancreatic tumor, overlapped with $\mathrm{T}_{2}$ mapping MRI of tumor, before and I week after the treatment. (B) Corresponding quantitative MR signal changes. Data are presented as mean $\pm S D(n=3)$. (C) Comparison of the averaged tumor weights from different treatment groups $(n=6)$ showed the significant inhibition of the tumor growth by ATF-CNIO-CDDP $(P<0.01)$ after once-weekly IV administration for 4 weeks, and $(D)$ the changes of relative body weight over the treatment course. $(E)$ Ex vivo $T_{2}$-weighted $M R$ images of tumors collected from mice after the 4-week treatment, and $(F)$ the corresponding $T_{2}$ relaxation time measured by MRI. $* * P<0.01$, $* * * P<0.001$.

Abbreviations: ATF, amino-terminal fragment; au, absorbance unit; CDDP, cisplatin; CNIO, milk protein (casein)-coated magnetic iron oxide; IV, intravenous; MRI, magnetic resonance imaging; ns, nonsignificant; SD, standard deviation; wk, week.

mice $(n=6)$ in comparison with PBS, nontargeted CNIOCDDP, and free CDDP. As shown in Figure 3C, ATFCNIO-CDDP exhibited superior antitumor activity compared to the other groups. The average tumor weight from the
ATF-CNIO-CDDP-treated group was $0.8 \pm 0.1 \mathrm{~g}$, compared with around $1.5 \mathrm{~g}$ for the other groups $(P<0.01)$. No significant tumor suppression evaluated by the tumor weight was observed for the groups treated with free CDDP and 
nontargeted CNIO-CDDP; however, histological studies revealed the inhibition of tumor metastasis in neighboring organs after the animals received either CDDP or CNIOCDDP or ATF-CNIO-CDDP, respectively (Figure S1). In addition, less body weight change was observed in mice treated with CDDP, CNIO-CDDP, or ATF-CNIO-CDDP than the control group treated with PBS (Figure 3D), which gained $17 \%$ of initial body weight due to the malignant ascites caused by the metastasis. Reduced anaphylaxis, such as skin irritation and ulceration, was observed in the mice that received CNIO-CDDP or ATF-CNIO-CDDP, compared with those that received free CDDP, suggesting the protection and slow release of CDDP with CNIO and ATF-CNIO nanocarriers. Necropsy analysis of organ tissues collected from the animals treated with ATF-CNIO-CDDP showed no detectable toxicity in liver and spleen tissues, while hepatocellular damage was observed in the mouse livers treated with free CDDP (Figure S2), indicating that the delivery of CDDP with CNIO nanocarriers potentially reduced the CDDP systemic toxicity at the same dosage used in conventional delivery methods.

Prolonged circulation time and improved drug accumulation in the tumor have been obtained by using polymer- and lipid-based nanocarriers, compared with free small-molecule drugs. ${ }^{33,34}$ Given the hydrodynamic size of $\sim 30 \mathrm{~nm}$, ATFCNIO-CDDP can take advantage of the prolonged blood circulation half life time ( $t_{1 / 2} \sim 4.2$ hours), allowing sufficient time to reach the targeted tumor, thus enhancing tumor accumulation via the enhanced permeability and retention (EPR) effect. The lower antitumor effect of free CDDP in the orthotopic pancreatic cancer model here can be explained by the fact that free CDDP has a much shorter blood circulation time and faster clearance, thus it fails to maintain a sufficient plasma level and gradient that can drive the drug to the tumor. Furthermore, the developed ATF-CNIO-CDDP demonstrated sustained release of CDDP, which potentially ensures the effective release inside a tumor after accumulation. All those favorable conditions may contribute to the observed better delivery of CDDP by the CNIO nanoparticle drug delivery system.

Pancreatic tumors are noted for the abundant stromal structures and poorly developed vasculature, impairing the therapeutic efficiency. ${ }^{35-38}$ Among the cancer biomarkers, UPAR is known to be overexpressed in both tumor cells and stromal structures. ATF has been demonstrated to be able to target uPAR-expressing cancer cells when being used as the targeting ligand conjugated on nanoparticle drug carriers. ${ }^{25,26}$ Therefore, this targeting approach is expected to promote the delivery of ATF-CNIO-CDDP into pancreatic tumors, resulting in higher accumulation of ATF-CNIO-CDDP, which benefits from the greater cellular uptake via receptor-mediated endocytosis. ${ }^{39,40}$ Earlier studies have proved that receptor-mediated targeting can enhance tumor accumulation rather than the EPR-effect-mediated delivery. ${ }^{41,42}$ Here in this study, nontargeted CNIO-CDDP failed at improving the treatment efficacy in vivo due to the insufficient drug concentration inside the tumor, although these nontargeted CNIO-CDDP showed efficient tumor cell killing activity in vitro. In contrast, ATF-CNIO-CDDP showed potential against drug resistance for pancreatic cancer treatment by improving tumor targeting and tumor retention, as well as sustained- and $\mathrm{pH}$-facilitated-release at the tumor site. Consequently, the results from the current study further demonstrate that active targeting can enhance therapeutic efficacy over passive targeting.

\section{Ex vivo and histological validations of targeted delivery of ATF-CNIO-CDDP in tumors}

The improved delivery of ATF-CNIO-CDDP in tumors was further confirmed by ex vivo MRI measurement of the $\mathrm{T}_{2}$ relaxation time of tumor tissues. As shown in Figure 3E, ATF-CNIO-CDDP-treated tumors exhibited much darker $\mathrm{T}_{2}$ signals (ie, shorter $\mathrm{T}_{2}$ relaxation times) than those that received nontargeted CNIO-CDDP, indicating much higher accumulation of ATF-CNIO-CDDP in tumors. The average transverse $T_{2}$ relaxation time, which is known to be dependent on the iron concentration, ${ }^{24}$ was determined to be $44.8 \mathrm{~ms}$ for the ATF-CNIO-CDDP-treated tumors, which is about half of the $\mathrm{T}_{2}$ for the tumors treated with CNIO-CDDP (77.8 ms), and one-third of that for the tumors treated with free CDDP and PBS (154.0, $159.3 \mathrm{~ms}$, respectively) (Figure 3F).

To validate the targeted delivery of ATF-CNIO-CDDP, Prussian blue staining for Fe and immunofluorescence labeling of UPAR were performed on the collected tumor sections. ATF-CNIO-CDDP was found to be colocalized well with areas that express high levels of uPAR (Figure 4). In addition, more intense Prussian blue stains were observed in the tumor sections from the ATF-CNIO-CDDP-treated group compared to other groups, further confirming the higher accumulation of ATF-CNIO-CDDP in tumors. To investigate the effect of the tumor microenvironment on delivery, we particularly examined collagen fibers, which are the main component of tumor stroma, with haematoxylin and eosin (H\&E) and Sirius red staining. The results from Sirius red staining in which collagen was stained in red showed that there was a 

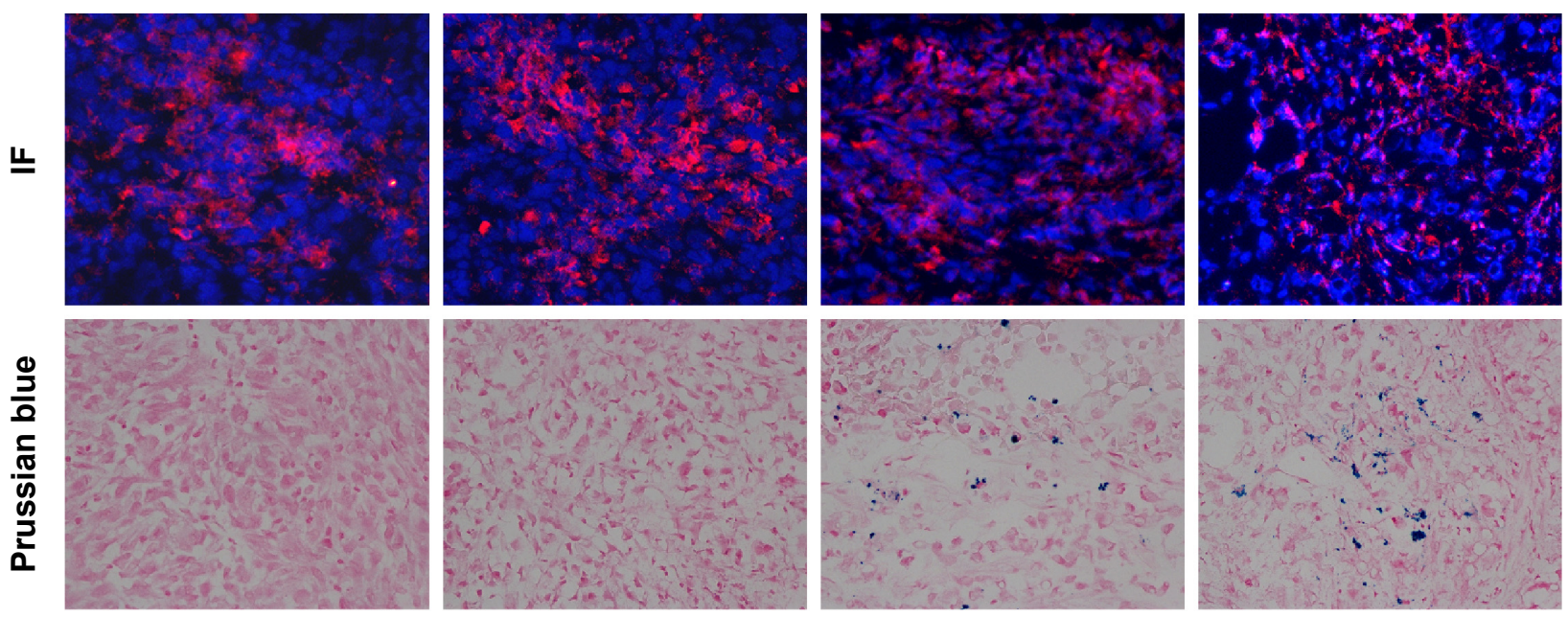

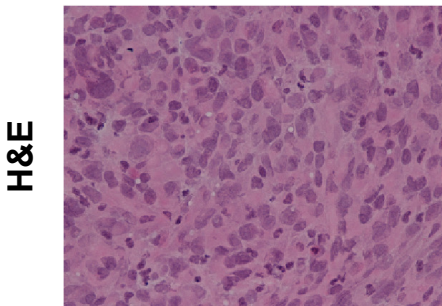

Control

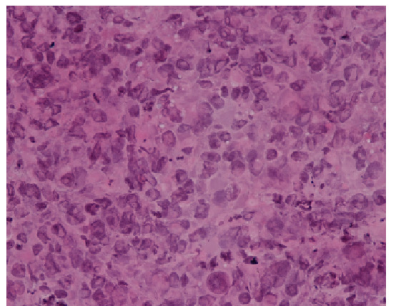

Free CDDP

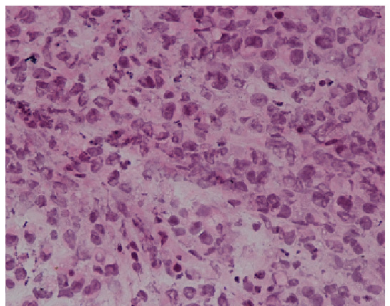

CNIO-CDDP

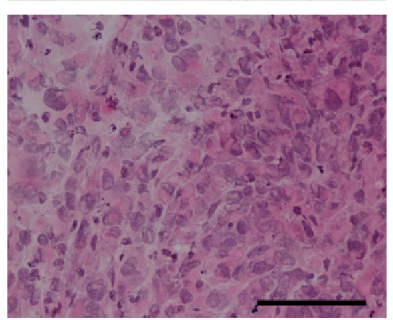

ATF-CNIO-CDDP

Figure 4 Histological validation of nanoparticle accumulation in orthotopic MIA PaCa-2 tumors with IF labeling of uPAR, Prussian blue for iron, and H\&E staining.

Notes: Heterogeneous expression of uPAR (in red) was detected in MIA PaCa-2 tumors, where nuclei were stained with DAPI (in blue). Prussian blue staining revealed the area accumulated with targeted ATF-CNIO-CDDP overlapped with the area showing uPAR overexpression. The scale bar is $50 \mu \mathrm{m}$.

Abbreviations: ATF, amino-terminal fragment; CDDP, cisplatin; CNIO, milk protein (casein)-coated magnetic iron oxide; $\mathrm{H \& E}$, hemtoxylin and eosin; IF, immunofluorescence; uPAR, urokinase plasminogen activator receptor; DAPI, 4',6-diamidino-2-phenylindole.

decrease in the amount of collagen bundles in tumors after the treatment with ATF-CNIO-CDDP when compared to the tumors that received other treatments (Figure 5). Although the mechanism and effect of the ATF-CNIO-CDDP treatment on the tumor microenvironment remain to be fully investigated, the decrease of collagen bundles may indicate the disruption of the tumor stroma, thus leading to more efficient delivery of the ATF-CNIO-CDDP and improved treatment. ${ }^{43}$

\section{Conclusion}

A new class of multifunctional nanotheranostics that combines imaging capability (ie, MRI), tumor targeting,
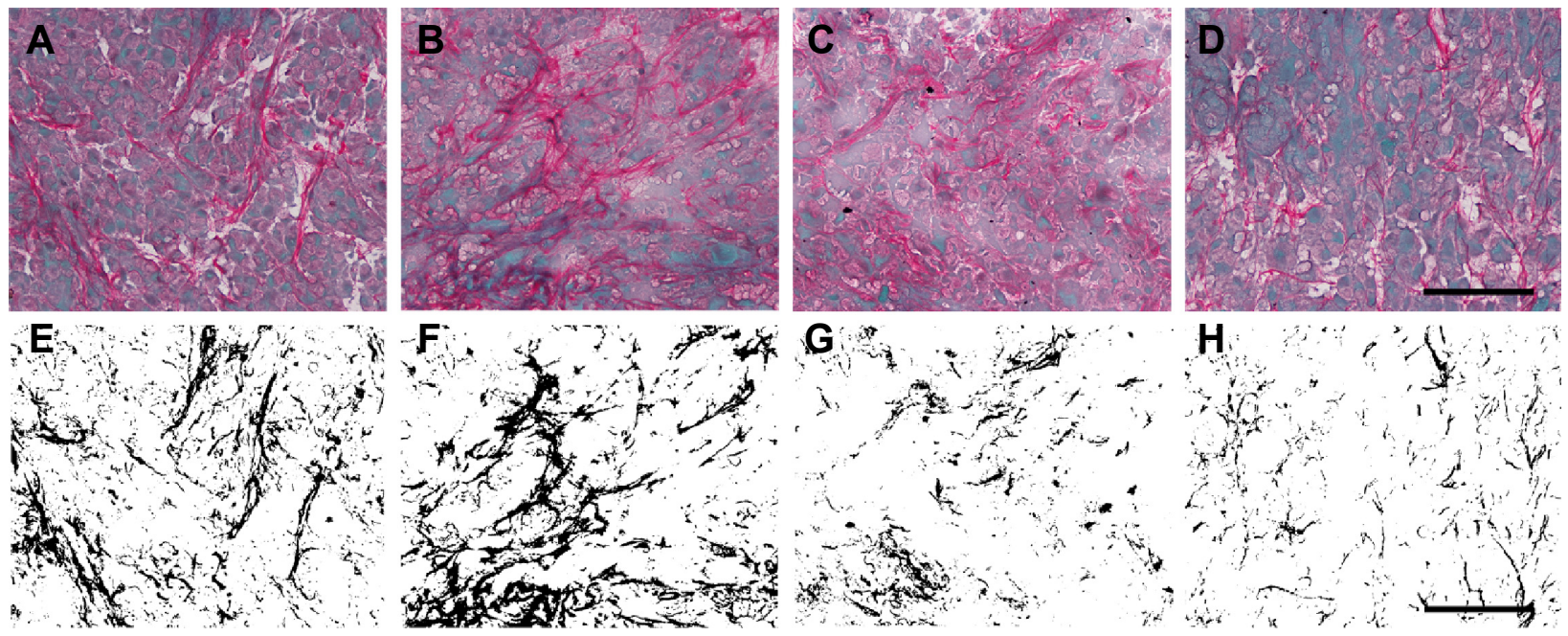

Figure 5 (Continued) 


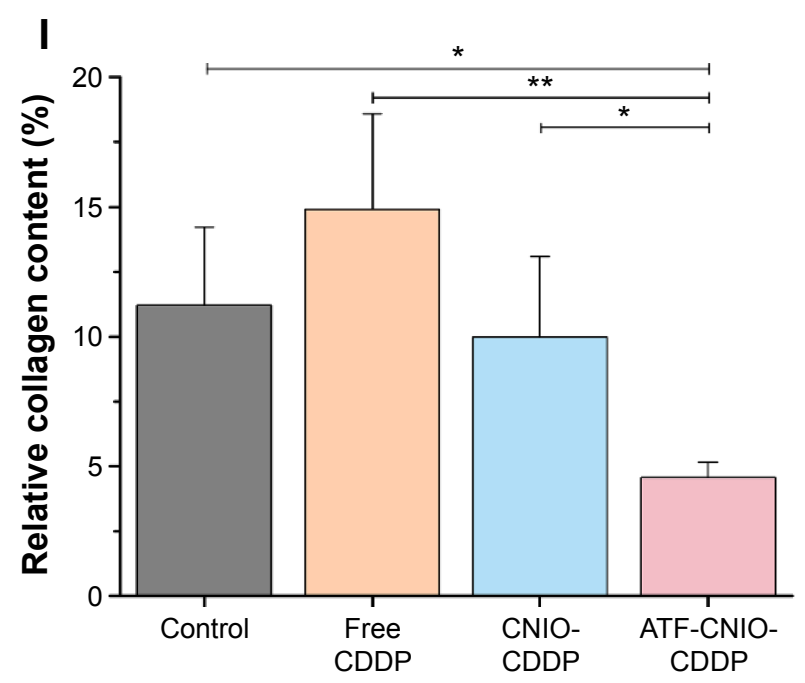

Figure 5 Sirius red staining and quantitative analysis of collagen fiber bundle of orthotopic MIA PaCa-2 tumors after treatment.

Notes: (A-D) Representative Sirius red staining of tumor sections from treated and untreated groups and (E-H) the extracted collagen bundles from corresponding images, which revealed fewer collagen bundles in ATF-CNIO-CDDP treated tumors. The scale bar is $50 \mu \mathrm{m}$. (I) Relative collagen contents of tumors treated with different CDDP formulations which were estimated based on analysis of semiautomatic segmentation of the collagen bundles stained from corresponding images of Sirius red stained tumor sections. $* P<0.05, * * P<0.01$.

Abbreviations: ATF, amino-terminal fragment; CDDP, cisplatin; CNIO, milk protein (casein)-coated magnetic iron oxide.

and drug delivery has been developed with milk protein (casein)-coated iron oxide nanoparticles. With the ATF ligand, ATF-CNIO-CDDP nanoparticles actively targeted uPAR-overexpressed pancreatic tumor and tumor stroma in vivo, promoting sufficient tumor-targeted delivery of CDDP. The sustained- and low-pH-facilitated release of CDDP following the improved accumulation in tumor facilitated the antitumor activity. Subsequently, ATF-CNIO-CDDP demonstrated improved tumor inhibition and reduced side effects in orthotopic pancreatic tumor mouse models, exhibiting high relevance to clinical research. Furthermore, the delivery of active drugs by these nanovehicles could be monitored by MRI in vivo. As such, milk-protein-coated magnetic nanoparticles may offer a promising platform for image-guided and targeted delivery in cancer treatment.

\section{Acknowledgment}

This work is supported by NIH R01CA154846-02 (HM and LY), NCI's Cancer Nanotechnology Platform Project (CNPP) grant (U01CA151810-02 to LY and HM) and Emory Molecular Translational Imaging Center of NCI's in vivo Cellular and Molecular Imaging Center grant (ICMIC, P50CA128301-01A10003 to HM and LY).

\section{Disclosure}

The authors report no conflicts of interest in this work.

\section{References}

1. Hidalgo M. Pancreatic cancer. N Engl J Med. 2010;362(17): $1605-1617$.
2. Siegel R, Ma J, Zou Z, Jemal A. Cancer statistics, 2014. CA Cancer J Clin. 2014;64(1):9-29.

3. Conroy T, Desseigne F, Ychou M, et al. FOLFIRINOX versus gemcitabine for metastatic pancreatic cancer. $N$ Engl J Med. 2011;364(19): $1817-1825$.

4. De Jesus-Acosta A, Oliver GR, Blackford A, et al. A multicenter analysis of GTX chemotherapy in patients with locally advanced and metastatic pancreatic adenocarcinoma. Cancer Chemother Pharmacol. 2012;69(2):415-424.

5. Von Hoff DD, Ervin T, Arena FP, et al. Increased survival in pancreatic cancer with nab-paclitaxel plus gemcitabine. $N$ Engl J Med. 2013; 369(18):1691-1703.

6. Lau JK, Deubel VD. Loss of ammine from platinum(II) complexes: implications for cisplatin inactivation, storage, and resistance. Chemistry. 2005;11:2849-2855.

7. Liu D, Poon C, Lu K, He C, Lin W. Self-assembled nanoscale coordination polymers with trigger release properties for effective anticancer therapy. Nat Commun. 2014;5:4182.

8. Maksimenko A, Dosio F, Mougin J, et al. A unique squalenoylated and nonpegylated doxorubicin nanomedicine with systemic longcirculating properties and anticancer activity. Proc Natl Acad Sci U S A. 2014;111(2):E217-E226.

9. Nahire R, Haldar MK, Paul S, et al. Multifunctional polymersomes for cytosolic delivery of gemcitabine and doxorubicin to cancer cells. Biomaterials. 2014;35(24):6482-6497.

10. Zhao $\mathrm{P}$, Zheng $\mathrm{M}$, Yue $\mathrm{C}$, et al. Improving drug accumulation and photothermal efficacy in tumor depending on size of ICG loaded lipidpolymer nanoparticles. Biomaterials. 2014;35(23):6037-6046.

11. Al-Hajeili M, Azmi AS, Choi M. Nab-paclitaxel: potential for the treatment of advanced pancreatic cancer. Onco Targets Ther. 2014;7: 187-192.

12. Burger KN, Staffhorst RW, de Vijlder HC, et al. Nanocapsules: lipidcoated aggregates of cisplatin with high cytotoxicity. Nat Med. 2002; $8(1): 81-84$

13. Hall MD, Okabe M, Shen DW, Liang XJ, Gottesman MM. The role of cellular accumulation in determining sensitivity to platinum-based chemotherapy. Annu Rev Pharmacool Toxicol. 2008;48:495-535.

14. Doane TL, Burda C. The unique role of nanoparticles in nanomedicine: imaging, drug delivery and therapy. Chem Soc Rev. 2012;41(7): 2885-2911. 
15. Murakami M, Cabral H, Matsumoto Y, et al. Improving drug potency and efficacy by nanocarrier-mediated subcellular targeting. Sci Transl Med. 2011;3(64):64ra2.

16. Timko BP, Dvir T, Kohane DS. Remotely triggerable drug delivery systems. Adv Mater. 2010;22(44):4925-4943.

17. Huang J, Wang L, Lin R, et al. Casein-coated iron oxide nanoparticles for high mri contrast enhancement and efficient cell targeting. ACS Appl Mater Interfaces. 2013;5(11):4632-4639.

18. Guo P, Huang J, Wang L, et al. ICAM-1 as a molecular target for triple negative breast cancer. Proc Natl Acad Sci U S A. 2014;111(41): $14710-14715$

19. Zhen X, Wang X, Xie C, Wu W, Jiang X. Cellular uptake, antitumor response and tumor penetration of cisplatin-loaded milk protein nanoparticles. Biomaterials. 2013;34(4):1372-1382.

20. Lee GY, Qian WP, Wang L, et al. Theranostic nanoparticles with controlled release of gemcitabine for targeted therapy and MRI of pancreatic cancer. ACS Nano. 2013;7(3):2078-2089.

21. Golla ED, Ayres GH. Spectrophotometric determination of platinium with ortho-phenylenediamine. Talanta. 1973;20(2):199-210.

22. Huang J, Wang L, Zhong X, Li Y, Yang L, Mao H. Facile nonhydrothermal synthesis of oligosaccharide coated sub-5 nm magnetic iron oxide nanoparticles with dual MRI contrast enhancement effects. J Mater Chem B. 2014;2(33):5344-5351.

23. Canbay A, Guicciardi ME, Higuchi H, et al. Cathepsin B inactivation attenuates hepatic injury and fibrosis during cholestasis. J Clin Invest. 2003;112(2):152-159.

24. Huang J, Zhong X, Wang L, Yang L, Mao H. Improving the magnetic resonance imaging contrast and detection methods with engineered magnetic nanoparticles. Theranostics. 2012;2(1):86-102.

25. Yang L, Mao H, Cao Z, et al. Molecular imaging of pancreatic cancer in an animal model using targeted multifunctional nanoparticles. Gastroenterology. 2009;136(5):1514-1525.

26. Yang L, Sajja HK, Cao Z, et al. uPAR-targeted optical imaging contrasts as theranostic agents for tumor margin detection. Theranostics. 2014;4(1):106-118.

27. Nishiyama N, Okazaki S, Cabral H, et al. Novel cisplatin-incorporated polymeric micelles can eradicate solid tumors in mice. Cancer Res. 2003; 63(24):8977-8983.

28. Peng X-H, Wang Y, Huang D, et al. Targeted delivery of cisplatin to lung cancer using ScFvEGFR-heparin-cisplatin nanoparticles. ACS Nano. 2011;5(12):9480-9493.

29. Kettering M, Zorn H, Bremer-Streck S, et al. Characterization of iron oxide nanoparticles adsorbed with cisplatin for biomedical applications. Phys Med Biol. 2009;54(17):5109-5121.
30. Unterweger H, Tietze R, Janko C, et al. Development and characterization of magnetic iron oxide nanoparticles with a cisplatin-bearing polymer coating for targeted drug delivery. Int J Nanomedicine. 2014; 9:3659-3676.

31. Todd RC, Lovejoy KS, Lippard SJ. Understanding the effect of carbonate ion on cisplatin binding to DNA. J Am Chem Soc. 2007;129(20): 6370-6371.

32. Uchino H, Matsumura Y, Negishi T, et al. Cisplatin-incorporating polymeric micelles (NC-6004) can reduce nephrotoxicity and neurotoxicity of cisplatin in rats. Br J Cancer. 2005;93(6):678-687.

33. Guo S, Wang Y, Miao L, et al. Lipid-coated cisplatin nanoparticles induce neighboring effect and exhibit enhanced anticancer efficacy. ACS Nano. 2013;7(11):9896-9904.

34. Cabral H, Murakami M, Hojo H, et al. Targeted therapy of spontaneous murine pancreatic tumors by polymeric micelles prolongs survival and prevents peritoneal metastasis. Proc Natl Acad Sci U S A. 2013; 110(28):11397-11402.

35. Wang Z, Li Y, Ahmad A, et al. Pancreatic cancer: understanding and overcoming chemoresistance. Nat Rev Gastroenterol Hepatol. 2011;8(1): 27-33.

36. Oettle H. Progress in the knowledge and treatment of advanced pancreatic cancer: from benchside to bedside. Cancer Treat Rev. 2014; 40(9):1039-1047.

37. Guengoer C, Hofmann BT, Wolters-Eisfeld G, Bockhorn M. Pancreatic cancer. Br J Pharmacol. 2014;171(4):849-858.

38. Lunardi S, Muschel RJ, Brunner TB. The stromal compartments in pancreatic cancer: are there any therapeutic targets? Cancer Lett. 2014; 343(2):147-155.

39. Wang M, Thanou M. Targeting nanoparticles to cancer. Pharm Res 2010;62(2):90-99.

40. Canton I, Battaglia G. Endocytosis at the nanoscale. Chem Soc Rev. 2012;41(7):2718-2739.

41. Schleich N, Po C, Jacobs D, et al. Comparison of active, passive and magnetic targeting to tumors of multifunctional paclitaxel/SPIO-loaded nanoparticles for tumor imaging and therapy. J Control Release. 2014; 194:82-91.

42. Sykes EA, Chen J, Zheng G, Chan WC. Investigating the impact of nanoparticle size on active and passive tumor targeting efficiency. ACS Nano. 2014;8(6):5696-5706.

43. Alvarez R, Musteanu M, Garcia-Garcia E, et al. Stromal disrupting effects of nab-paclitaxel in pancreatic cancer. Br J Cancer. 2013;109(4): 926-933. 


\section{Supplementary materials}
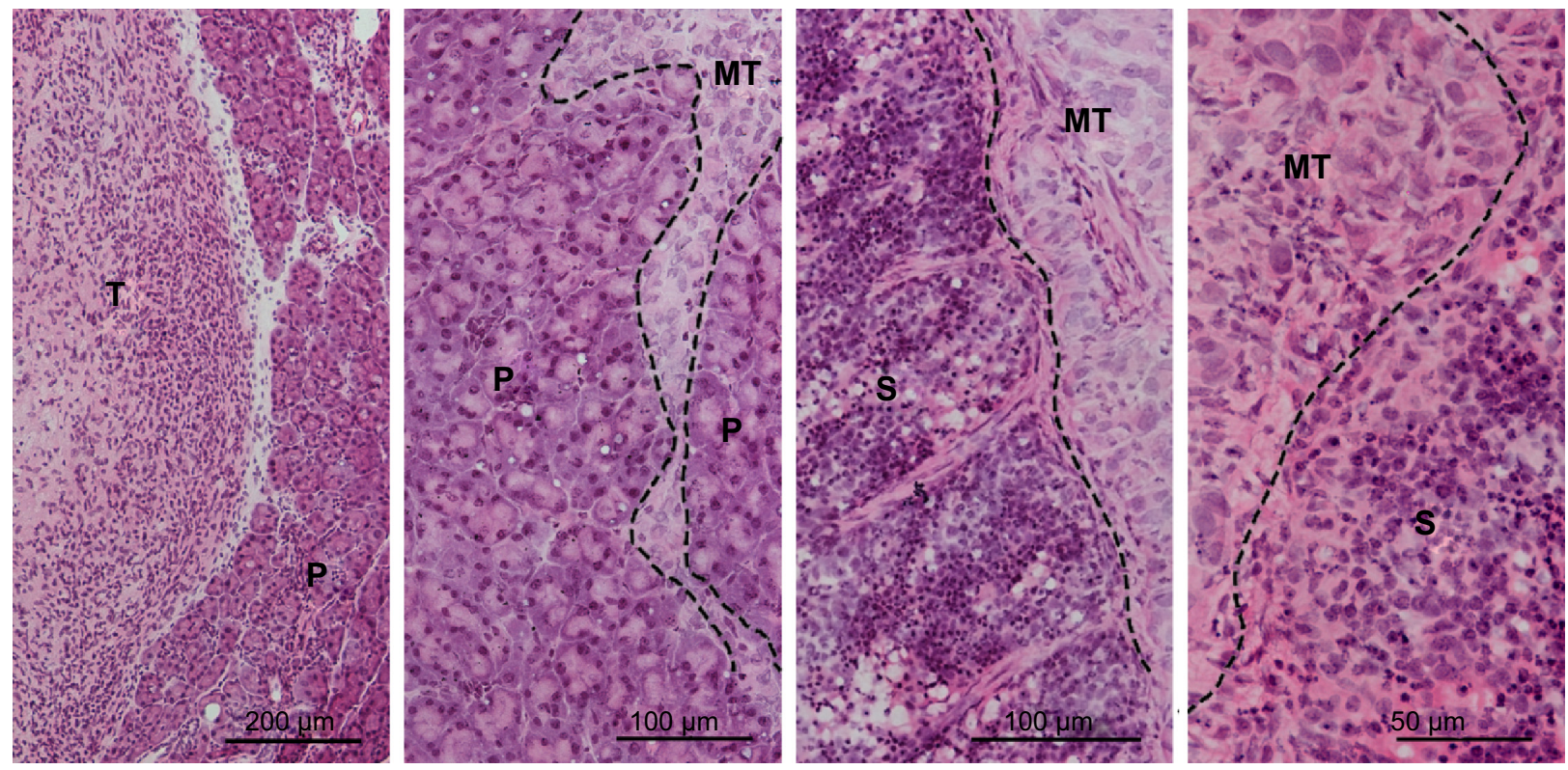

Figure SI Representative H\&E staining of initial and metastatic tumors from the group treated with PBS only.

Notes: Most of the tumors (T) have obvious boundary along with pancreas (P) (left first panel), while metastatic tumors (MT) were observed invading into deeper pancreas (P) or spleen (S). No such metastasis was observed in the groups treated with free CDDP, CNIO-CDDP, or ATF-CNIO-CDDP.

Abbreviations: ATF, amino-terminal fragment; CDDP, cisplatin; CNIO, milk protein (casein)-coated magnetic iron oxide; $\mathrm{H \& E}$, hematoxylin and eosin; PBS, phosphate-buffered saline. 


\section{Sirius red}
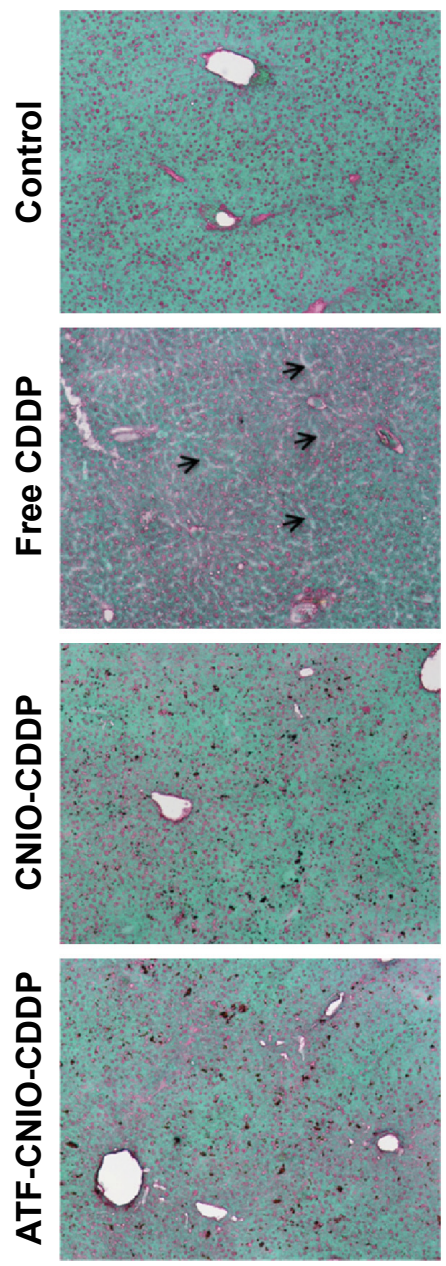

Liver
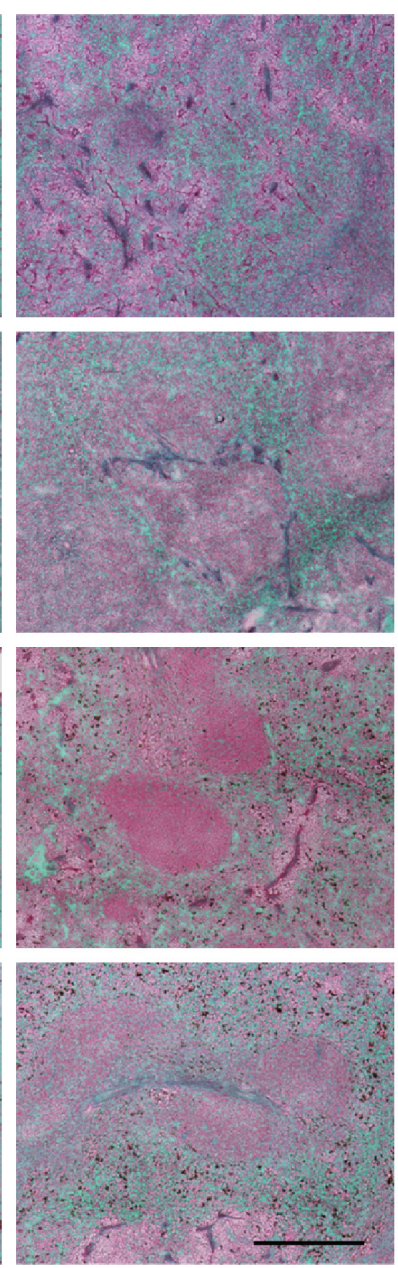

Spleen
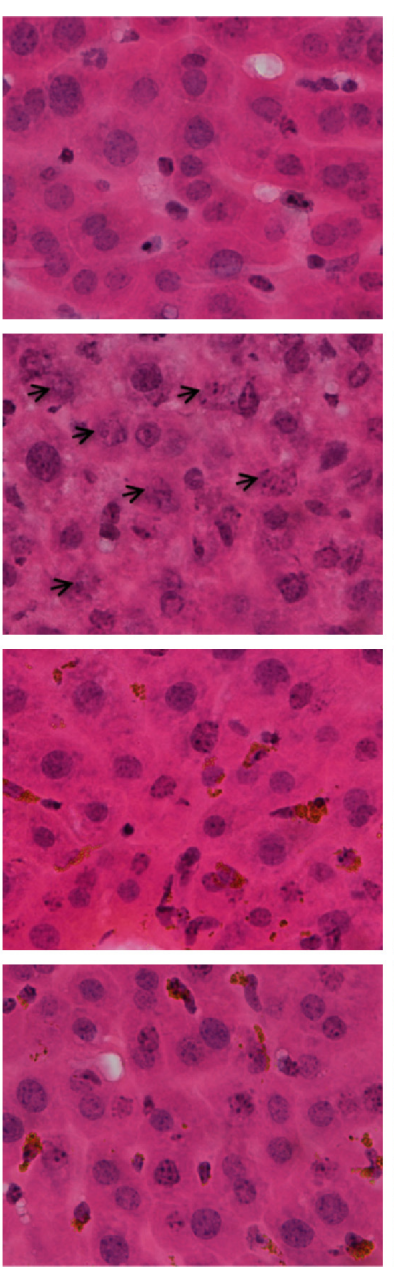

Liver
H\&E
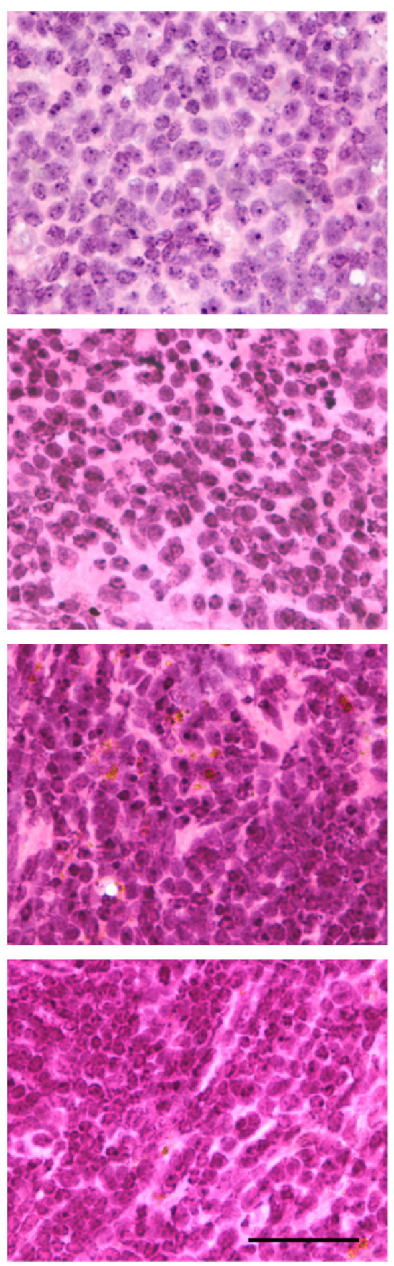

Spleen

Figure S2 Representative Sirius red and H\&E staining of liver and spleen sections.

Notes: As indicated by black arrows, hepatic degeneration and vacuolization with necrotic cells were observed in the group that received free CDDP, while little damage was observed in the groups that received CNIO-CDDP and ATF-CNIO-CDDP, demonstrating reduced systemic toxicity to normal tissues by nanocarriers. The scale bar is 100 and $50 \mu \mathrm{m}$, respectively.

Abbreviations: ATF, amino-terminal fragment; CDDP, cisplatin; CNIO, milk protein (casein)-coated magnetic iron oxide; H\&E, hematoxylin and eosin.

International Journal of Nanomedicine

\section{Publish your work in this journal}

The International Journal of Nanomedicine is an international, peerreviewed journal focusing on the application of nanotechnology in diagnostics, therapeutics, and drug delivery systems throughout the biomedical field. This journal is indexed on PubMed Central, MedLine, CAS, SciSearch ${ }^{\circledR}$, Current Contents ${ }^{\circledR} /$ Clinical Medicine,

\section{Dovepress}

Journal Citation Reports/Science Edition, EMBase, Scopus and the Elsevier Bibliographic databases. The manuscript management system is completely online and includes a very quick and fair peer-review system, which is all easy to use. Visit http://www.dovepress.com/ testimonials.php to read real quotes from published authors.

\footnotetext{
Submit your manuscript here: http://www.dovepress.com/international-journal-of-nanomedicine-journal
} 